TRANSACTIONS OF THE

AMERICAN MATHEMATICAL SOCIETY

Volume 351, Number 12, Pages 4837-4846

S 0002-9947(99)02238-2

Article electronically published on May 26, 1999

\title{
HOMOLOGY OF THE UNIVERSAL COVERING OF A CO-H-SPACE
}

\author{
NORIO IWASE, SHIROSHI SAITO, AND TOSHIO SUMI
}

\begin{abstract}
The problem 10 posed by Tudor Ganea is known as the Ganea conjecture on a co-H-space, a space with co-H-structure. Many efforts are devoted to show the Ganea conjecture under additional assumptions on the given co-H-structure. In this paper, we show a homological property of coH-spaces in a slightly general situation. As a corollary, we get the Ganea conjecture for spaces up to dimension 3.
\end{abstract}

\section{INTRODUCTION}

In this paper we work in the category of connected CW complexes with base points. A connected CW complex $X$ is called a co-H-space when the homotopy set $[X, Y]$ has a multiplication with two-sided unit which is natural with respect to $Y$, where we denote by $[K, L]$ the set of homotopy classes of base point preserving mappings from $K$ to $L$. We call the natural multiplication the co-H-structure. Ganea problem 10 is as follows: Does a connected space with co-H-structure have the homotopy type of a one-point-sum of a wedge sum of circles and a simply connected space?

It is known by S. Eilenberg and T. Ganea [5] that if a co-H-space $X$ is paracompact and normal, $B \pi_{1}(X)$ has the homotopy type of a wedge sum of circles, say $B$. Thus we have two mappings $i: B \rightarrow X$ and $j: X \rightarrow B$, where $i$ induces an isomorphism of the fundamental groups and $j$ is the classifying mapping of the universal covering $\tau: \tilde{X} \rightarrow X$. Clearly, we may choose the mappings so that $j i \simeq 1_{X}$, the identity, and hence, $B$ is a retract of $X$ up to homotopy.

Under the assumption, we have the cofibre $c: X \rightarrow C$ of $i: B \rightarrow X$. Then $c: X \rightarrow C$ has a homotopy splitting (see Theorem 3.3). By using the co-H-structure, one can easily get a canonical homology equivalence $X \rightarrow B \vee C$ which induces also an isomorphism of fundamental groups. These properties, however, would not guarantee that the two spaces have the same homotopy type (see G.W. Whitehead [16] pp.183-184).

Berstein and Dror [2] showed that the conjecture is true provided that the coaction induced from the co-H-structure is associative. Hilton, Mislin and Roitberg [9] showed that the conjecture is true provided that the co-H-structure gives a natural algebraic loop structure on the homotopy sets $[X$,$] . In addition, we can$

Received by the editors May 13, 1997.

1991 Mathematics Subject Classification. Primary 55P45, Secondary 19A13.

Key words and phrases. LS category, co-H-space, deck transformation.

The first author's research was supported by Grant-in-Aid for Scientific Research (C)08640125 from the Ministry of Education, Science, Sports and Culture. 
easily see that two conditions given by the above authors are valid for all co- $\mathrm{H}$ spaces if the Ganea conjecture is true.

Our approach is completely different. In this paper, we use the existence of the co-H-structure for a co-H-space $X$ of up to dimension 3 to deform the homotopy splitting: $C \rightarrow X$ to get a homotopy equivalence $B \vee C \rightarrow X$ in light of a theorem of Seshadri, Cohn and Bass on algebras. To proceed further, we need to exclude the Whitehead products from the boundary of the cells.

We should give a comment on the work of Dr. Komatsu on the theory of links [11]. In the proof of [11], we can find out an argument which gives a proof of Ganea's conjecture for co-H-spaces whose cohomology is concentrated in one dimension other than 1, using Fox's free differential calculus.

We thank M.G. Barratt and Takao Matumoto for their helpful comments on our earlier study of this subject.

\section{MAin Theorem}

A co-pairing introduced by Oda [12] is a mapping from a space $A$ to a space $B \vee C$, whose projections to $B$ and $C$ are called its co-axes. Let us introduce a notion of co-action: a co-action of $B$ under $A$ along $f: A \rightarrow B$ is a co-pairing $\mu: A \rightarrow B \vee A$ with co-axes $f$ and the identity $1_{A}$.

Our main theorem is as follows:

Theorem 2.1. Let $X$ be a finite $C W$ complex and $j: X \rightarrow B$ the classifying mapping of the universal covering $\tau: \tilde{X} \rightarrow X$. If there exists a co-action of $B$ under $X$ along $j$, then $H_{*}(\tilde{X} ; \mathbb{Z}) \cong \mathbb{Z} \pi \otimes H_{*}(X ; \mathbb{Z}), \pi=\pi_{1}(X)$ for $*>1$.

Corollary 2.2. Let $X$ be a finite co-H-space. If $H_{n+1}(X ; \mathbb{Z})$ is concentrated in dimensions $1, n$ and $n+1$ for $1<n$ with $H_{n+1}(X ; \mathbb{Z})$ torsion free, then $X$ splits into a one-point sum of $S^{1}$ 's, $S^{n}$ 's, $S^{n+1}$ 's and $S^{n}(m)$ 's, where we denote by $S^{k}(\ell)$ the Moore space $S^{k} \cup_{\ell_{k}} e^{k+1}$.

Hence, the Ganea conjecture is true for complexes whose homology is concentrated in dimensions $\leq 3$.

The key lemma of the theorem is based on the work done by Seshadri [15], Cohn [3] and Bass [1], that every finitely generated projective $A$-module is free when $A$ is a $f i r$, a free ideal ring.

The algebraic version of the Ganea conjecture could be described as follows.

Conjecture 2.3. Let $A$ be an algebra over a principal ideal domain $R$. If an $A$ module $P$ is a direct summand of an induced $A$-module $A \otimes_{R} M$ from an $R$-module $M$, then $P$ itself is induced.

We do not know anything about the general case but for the case when $R=\mathbb{Z}$, the ring of integers, $A$ is the group ring of a free group over $\mathbb{Z}$ and $P$ is finitely generated.

From now on, we reserve the symbol $X$ for a path-connected CW complex of finite type, $\pi$ for its fundamental group, $j: X \rightarrow B \pi$ the classifying mapping of the universal covering $\tau: \tilde{X} \rightarrow X$. Let us denote by $H_{q}(-)$ the $q$-th (ordinary) integral homology group and by $\pi_{q}(-)$ the $q$-th homotopy group for $q \geq 0$.

\section{Preliminaries}

First, we recall the following well-known result (by Ganea [7]). 
Fact 3.1. The following three conditions are equivalent for a $C W$ complex $W$ :

i) $W$ is a co-H-space.

ii) $L S$-cat $(W) \leq 1$, i.e., there is a mapping $W \rightarrow W \vee W$ so that the composition with the first and second projections $W \vee W \rightarrow W$ are homotopic to the identity.

iii) The evaluation $\Sigma \Omega W \rightarrow W$ has a homotopy section.

Let us call a group a co-H-group if the group, say $G$, admits a homomorphism $G \rightarrow G * G$ so that the compositions with the first and second projections $G *$ $G \rightarrow G$ are the identity of $G$, where we denote by $G * G$ the free product. Then the fundamental group of a co-H-space is clearly a co-H-group. Let us recall the following

Proposition 3.2 (Kan [10], Eilenberg-Ganea [5]). For $G$ a (discrete) group, the following four conditions are equivalent.

i) The classifying space $B G$ is a co-H-space.

ii) $G$ is a co-H-group.

iii) $G$ is a free group.

iv) BG has the homotopy type of a wedge sum of circles.

Proof. By Van Kampen's Theorem, $B G \vee B G$ has the homotopy type of $B(G * G)$. Hence, i) is equivalent to ii). And also iii) is equivalent to iv), because $B \mathbb{Z}$ has the homotopy type of a circle. Here, iv) implies i), since a circle is a co-H-space.

So we are left to show that i) implies iii). It is obtained by Proposition 3 in [5], when $B G$ is paracompact and normal. Here, let us give another simple proof of the implication as follows: Since $B G$ is a co-H-space, $\Sigma \Omega B G$ dominates $B G$ by Fact 3.1. Since $\Sigma \Omega B G \simeq \Sigma G$ has the homotopy type of a wedge sum of circles, its fundamental group is a free group, which could have infinitely many generators. Hence $G$ is a subgroup of a free group, and is itself a free group (see Crowell-Fox [4]). Thus i) implies iii) and this completes the proof of the proposition.

Hence by Proposition 3.2, the fundamental group $\pi$ is a free group and the classifying space $B \pi$ has the homotopy type of a wedge sum of circles, say $B$, if there exists a co-action of $B \pi$ under $X$ along $j$. From now on, we always assume the existence of such co-action and denote by $i: B \rightarrow X$ a mapping representing the generators of the fundamental group of $X$ and by $j: X \rightarrow B \pi \simeq B$ the classifying mapping of the universal covering $\tau: \tilde{X} \rightarrow X$, so that $j i$ is a homotopy equivalence. Hence we may assume that $j i \simeq 1_{B}$. Let us denote by $X \rightarrow C$ the mapping cone of $i: B \rightarrow X$. Under the above notations, we have the following

Theorem 3.3. There is a natural mapping $p: B \vee D(X) \rightarrow X$ with a (homotopy) section $s: X \rightarrow B \vee D(X)$, for some simply connected space $D(X)$.

Proof. Let $E$ be the homotopy pull-back of a mapping $\Delta_{j}=\left(j \times 1_{X}\right) \Delta_{X}: X \rightarrow$ $B \times X$ and the inclusion $k: B \vee X \rightarrow B \times X$ :

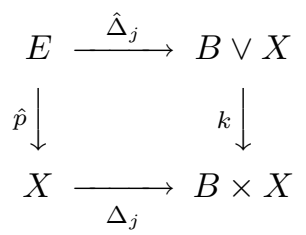

where $E$ can be described as the set $\left\{\left(x, \ell_{B}, \ell_{X}\right) \mid \ell_{B}: I \rightarrow B, \ell_{X}: I \rightarrow X, \ell_{B}(0)=\right.$ $\left.j(x), \ell_{X}(0)=x,\left(\ell_{B}(1), \ell_{X}(1)\right) \in B \vee X\right\}$. 


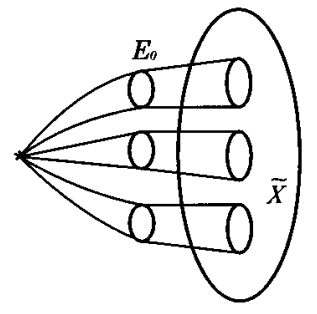

Figure 1

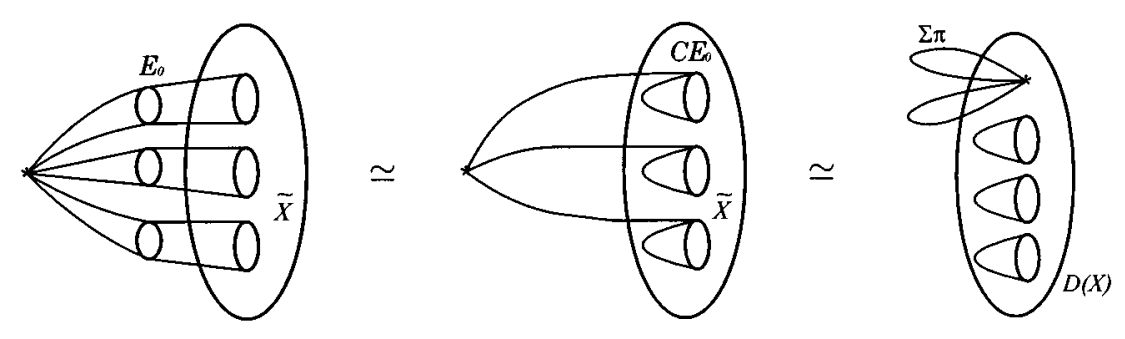

FIGURE 2

We remark here that the presence of a co-action of $B$ under $X$ along $j$ implies the existence of the homotopy section of $\hat{p}$.

We can find out subspaces $E_{1}, E_{2}$ and $E_{0}$ in $E$ as follows:

$$
\begin{aligned}
E_{1}=\left\{\left(x, \ell_{B}, \ell_{X}\right) \in\right. & \left.E \mid \ell_{X}(1)=*\right\} \\
& \supset\left\{\left(x, c(j(x)), \ell_{X}\right) \in E \mid \ell_{X}(1)=*\right\} \cong F^{1_{X}}, \\
E_{2}=\left\{\left(x, \ell_{B}, \ell_{X}\right) \in\right. & \left.E \mid \ell_{B}(1)=*\right\} \\
& \supset\left\{\left(x, \ell_{B}, c(x)\right) \in E \mid \ell_{B}(1)=*\right\} \cong F^{j} \text { and } \\
E_{0}=\left\{\left(x, \ell_{B}, \ell_{X}\right) \in\right. & \left.E \mid \ell_{B}(1)=*, \ell_{X}(1)=*\right\}=F^{\Delta_{j}}
\end{aligned}
$$

where $c(y)$ denotes the constant mapping at $y$ and $F^{f}$ denotes the homotopy fibre of $f$. Then we have $E=E_{1} \cup E_{2}$ and $E_{1} \cap E_{2}=E_{0}$. We can easily show that $F^{1 X} \simeq\{*\}$ and $F^{j} \simeq \tilde{X}$ are deformation retracts of $E_{1}$ and $E_{2}$, respectively. Hence $E$ has the homotopy type of the push-out $F^{1_{X}} \cup\left\{I \times E_{0}\right\} \cup F^{j} \simeq * \cup\left\{I \times E_{0}\right\} \cup \tilde{X}$, which is an unreduced mapping cone as shown in Figure 1.

Since $E_{0}$ is the homotopy fibre of $\Delta_{j}$, we have the following exact sequence of homotopy groups (sets):

$$
\cdots \rightarrow \pi_{1}(X)=\pi \stackrel{\Delta}{\rightarrow} \pi \times \pi=\pi_{1}(B \times X) \rightarrow \pi_{0}\left(E_{0}\right) \rightarrow * .
$$

Hence we have that the set $\pi_{0}\left(E_{0}\right)$ is in one-to-one correspondence with the group $\pi$.

Therefore $E_{0}$ is a topological sum $\coprod_{\omega \in \pi} E_{0}^{\omega}$ of connected component $E_{0}^{\omega}$ that corresponds to $\omega$. Then it follows that the unreduced mapping cone $\{*\} \cup$ $\left\{I \times E_{0}\right\} \cup \tilde{X}$ is naturally homotopy equivalent to $\Sigma \pi \vee D(X)$, where $D(X)$ is the reduced mapping cone of a mapping from $\bigvee_{\omega \in \pi} E_{0}^{\omega}$ to $\tilde{X}$. Since $\bigvee_{\omega \in \pi} E_{0}^{\omega}$ is path-connected and $\tilde{X}$ is 1-connected, we obtain by Van Kampen's Theorem that 
$D(X)$ is 1-connected and is natural with respect to $X$. Thus $E$ has the homotopy type of $\Sigma \pi \vee D(X)$, where $\Sigma \pi$ is a wedge sum of circles. (See Figure 2.)

Using the projection $\hat{p}: \Sigma \pi \vee D(X) \simeq E \rightarrow X$, we can define $p: B \vee D(X) \rightarrow X$ by $\left.p\right|_{B}=i$ and $\left.p\right|_{D(X)}=\left.\hat{p}\right|_{D(X)}$. Then by the definition of $D(X), p$ is natural with respect to $X$ up to homotopy.

A restriction of $\hat{p}$ to a circle gives an element of the fundamental group $\pi_{1}(X) \cong$ $\pi_{1}(B)$, and hence it factors through $i: B \rightarrow X$. Thus the restriction of $\hat{p}$ to $\Sigma \pi$ factors through $i: B \rightarrow X$. Thus the mapping $\hat{p}$ factors through $p: B \vee \tilde{X} \rightarrow X$ as $\hat{p}=p \hat{k}$ for some mapping $\hat{k}: E \simeq \Sigma \pi \vee D(X) \rightarrow B \vee \tilde{X}$.

On the other hand, $\hat{p}$ has a homotopy section $\hat{s}$, since $X$ admits a co-action of $B$. By putting $s=\hat{k} \hat{s}$, we see that $s$ is a homotopy section of $p$ :

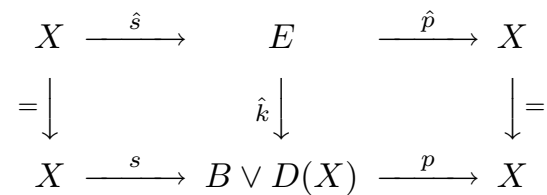

This implies the theorem.

By collapsing $B$ in $X$ and $B \vee D(X)$, we obtain the following

Corollary 3.4. $C$ is a retract of $D(X)$.

We fix the homotopy section $s$ of $p: B \vee D(X) \rightarrow X$. Let $\mu_{0}$ be the composition $\left(1_{B} \vee p\right)\left(\mu_{B} \vee 1_{D(X)}\right) s: X \rightarrow B \vee X$ where $\mu_{B}$ is the canonical co-multiplication of $B$ a wedge sum of circles. Thus we have the following

Corollary 3.5. If there exists a co-action of $B$ under $X$ along $j: X \rightarrow B$, then there is a co-action $\mu_{0}: X \rightarrow B \vee X$, such that the induced homomorphism $\mu_{0 *}$ : $\pi_{1}(X)=\pi \rightarrow \pi * \pi=\pi_{1}(B \vee X)$ is the standard (see Kan [10]) co-monoid structure of the free group $\pi$.

Let us consider the universal covering $\tau: \tilde{X} \rightarrow X$.

Corollary 3.6. For $q \geq 2$, there is a 1-connected finite complex $D_{q}$ such that $H_{q}(\tilde{X})$ is a direct summand of $Z \pi \otimes H_{q}\left(D_{q}\right)$.

Proof. By Theorem 3.3, $B \vee D(X)$ dominates $X: p s=1_{X}$. Since the $(q+1)$-skeleton $X^{(q+1)}$ of $X$ is a finite complex, the image $s\left(X^{(q+1)}\right)$ is in a finite subcomplex $B \vee D_{q}$ of $B \vee D(X)$ with $D_{q}$ 1-connected. On the other hand, $\left.p s\right|_{X^{(q+1)}}$ is homotopic to the canonical inclusion $X^{(q+1)} \rightarrow X$. Since the universal cover $\widetilde{B \vee D_{q}}$ of $B \vee D_{q}$ has the homotopy type of a wedge sum of $D_{q}$ 's indexed by $\pi, H_{q}(\tilde{X})$ is a direct summand of $H_{q}\left(\widetilde{B \vee D_{q}}\right) \cong Z \pi \otimes H_{q}\left(D_{q}\right)$.

\section{Proof of the Main Theorem}

Let us first consider the universal covering $\tau: \tilde{X} \rightarrow X$. Then the fundamental group $\pi=\langle\Lambda \mid\rangle$ acts on $\tilde{X}$, and hence on $H_{*}(\tilde{X})$, as a deck transformation group. The following is a generalization of Proposition 1.10 in [2].

\section{Proposition 4.1.}

$$
\tilde{H}_{q}(\tilde{X}) / \pi \cong \tilde{H}_{q}(C),
$$

where we denote by $H_{*}(\tilde{X}) / \pi$ the module $\mathbb{Z} \otimes_{\mathbb{Z} \pi} H_{*}(\tilde{X})$. 
Proof. By Theorem 3.3, there is a homotopy section $s$ of $p$ which induces the following commutative diagram up to homotopy.

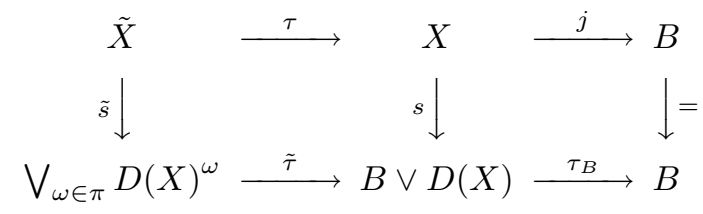

where $\tau_{B}$ is the first projection and $\tau$ and $\tilde{\tau}$ is the universal covering up to homotopy.

The $E^{1}$-terms of the Leray-Serre spectral sequence (see [16]) for the above fibrations $j$ and $\tau_{B}$ are isomorphic to the cellular chain complexes with the local coefficients in $H_{*}(\tilde{X})$ and $\mathbb{Z} \pi \otimes H_{*}(\tilde{X})$ given as

$$
\begin{aligned}
& E_{0, v}^{1}(j)=C_{0}\left(B, H_{v}(\tilde{X})\right) \cong H_{v}(\tilde{X}), \\
& E_{0, v}^{1}\left(\tau_{B}\right)=C_{0}\left(B, \mathbb{Z} \pi \otimes H_{v}(D(X))\right) \cong \mathbb{Z} \pi \otimes H_{v}(D(X)), \\
& E_{1, v}^{1}(j)=C_{1}\left(B, H_{v}(\tilde{X})\right) \cong \sum_{g \in \Lambda}\langle g\rangle H_{v}(\tilde{X}), \\
& E_{1, v}^{1}\left(\tau_{B}\right)=C_{1}\left(B, \mathbb{Z} \pi \otimes H_{v}(D(X))\right) \cong \sum_{g \in \Lambda}\langle g\rangle \mathbb{Z} \pi \otimes H_{v}(D(X)), \\
& E_{u, v}^{1}(j)=0, \quad \text { unless } 0 \leq u \leq 1, \\
& E_{u, v}^{1}\left(\tau_{B}\right)=0, \quad \text { unless } 0 \leq u \leq 1,
\end{aligned}
$$

where $v \geq 0, \Lambda$ the set of generators of $\pi$, and the first differentials $d^{1}(j)$ and $d^{1}\left(\tau_{B}\right): E_{1, v}^{1} \rightarrow E_{0, v}^{1}$ are given by the formulae

$$
\begin{aligned}
& d^{1}(j)(\langle g\rangle \tilde{x})=(g-1) \tilde{x}, \\
& d^{1}\left(\tau_{B}\right)(\langle g\rangle w)=(g-1) w
\end{aligned}
$$

where $\tilde{x} \in H_{v}(\tilde{X}), w \in \mathbb{Z} \pi \otimes H_{v}(\tilde{X})$ and $g \in \Lambda$. Since $E_{u, v}^{1}=0$ for $u \geq 2$, the spectral sequences collapse from the $E^{2}$-terms.

By comparing with the $E^{\infty}$-term, we obtain that $d^{1}\left(\tau_{B}\right)$ is injective. By Theorem $3.3, s$ induces a split monomorphism of $E^{1}$-terms, and hence $d^{1}(j)$ is also injective. Thus we obtain the following equations:

$$
\begin{aligned}
& E_{0, v}^{2}(j)=H_{v}(\tilde{X}) / \pi, \text { when } v \geq 0, \\
& E_{1, v}^{2}(j)=H_{v+1}(B), \text { when } v \geq 0, \\
& E_{u, v}^{2}(j)=0, \text { unless } 0 \leq u \leq 1 \text { or } v<0 .
\end{aligned}
$$

Since the spectral sequence for $j$ collapses, this implies the proposition.

Remark 4.2. The above proof also shows that the augmentation ideal $I$ of $\mathbb{Z} \pi$ can be described as the direct sum of $(g-1) \mathbb{Z} \pi$ 's indexed by $g \in \Lambda$, where each summand is the injection image of $\mathbb{Z} \pi$.

Lemma 4.3. Let $M$ be a $\mathbb{Z} \pi$-module which is a direct summand of an induced module from a finitely generated $\mathbb{Z}$-module. Then $M$ is itself an induced $\mathbb{Z} \pi$-module.

Assuming Lemma 4.3, we obtain the following 
Theorem 4.4. Let $X$ be a connected $C W$ complex of finite type. If $X$ admits a co-action of $B$ along $j$, we have the following isomorphism of $\mathbb{Z} \pi$-modules:

$$
\tilde{H}_{q}(\tilde{X})=\mathbb{Z} \pi \otimes G_{q} \quad \text { as } \mathbb{Z} \pi \text {-modules, }
$$

where $G_{q}$ is a submodule of $\tilde{H}_{q}(\tilde{X})$ isomorphic to $\tilde{H}_{q}(C)$. And the covering projection is equivalent to the canonical projection:

$$
\tilde{H}_{q}(\tilde{X})=\mathbb{Z} \pi \otimes G_{q} \rightarrow\left\{\mathbb{Z} \pi \otimes G_{q}\right\} / \pi \cong G_{q} \cong \tilde{H}_{q}(C) .
$$

Proof. By Corollary 3.6, it follows that $H_{q}(\tilde{X})$ is a direct summand of an induced module $\mathbb{Z} \pi \otimes H_{q}\left(D_{q}\right)$ from a finitely generated $\mathbb{Z}$-module $H_{q}\left(D_{q}\right)$. Then by Lemma 4.3 we obtain that $H_{q}(\tilde{X})$ is isomorphic with $\mathbb{Z} \pi \otimes G_{q}$ as $\mathbb{Z} \pi$-modules for some $\mathbb{Z}$-module $G_{q}$. Here the module $G_{q}$ is isomorphic with $\tilde{H}_{q}(C)$, by Proposition 4.1. The latter part is clear and this completes the proof of the theorem.

Since $H_{*}(X) \cong H_{*}(C)$ for $*>1$, Theorem 4.4 implies the main theorem. So we are left to show Lemma 4.3. First, let us consider the $p$-torsion part:

Proposition 4.5. Let $p$ be a prime and $M_{p}$ a $\mathbb{Z} \pi$-module which is a direct summand of an induced module from a finitely generated $p$-torsion module $F_{p}$. Then $M_{p}$ can be described as a direct sum: $M_{p} \cong M_{p}[1] \oplus M_{p}[2] \oplus \ldots \oplus M_{p}[h]$, where $M_{p}[i]$ is an induced $\mathbb{Z} \pi$-module from a free $\mathbb{Z} / p^{i} \mathbb{Z}$-module.

Proof. By the hypothesis, $M_{p}$ is a direct summand of $\mathbb{Z} \pi \otimes F_{p}$ and $F_{p}$ can be described as a direct sum $F_{p}[t] \oplus F_{p}[t+1] \oplus \ldots \oplus F_{p}[h], 1 \leq t \leq h$, where $F_{p}[i]$ is a free $\mathbb{Z} / p^{i} \mathbb{Z}$-module.

Let us introduce a notion of excess: $\operatorname{excess}\left(F_{p}\right)=2 h-t \geq 1$ if $F_{p}[t] \neq 0$ and $F_{p}[h] \neq 0$. We show the theorem by induction on excess: Let $k \geq 1$. By the induction hypothesis, we may suppose that we are done in case when $2 h-t \leq k-1$. First, let us assume that $h=t$. Then $F_{p}=F_{p}[h]$ is a free $\mathbb{Z} / p^{h} \mathbb{Z}$-module, $M_{p}$ is a projective $\left\{\mathbb{Z} / p^{h} \mathbb{Z}\right\} \pi$-module. Hence by Bass [1], Cohn [3] or Seshadri [15], $M_{p}$ is a free $\left\{\mathbb{Z} / p^{h} \mathbb{Z}\right\} \pi$-module. By putting $M_{p}[h]=M_{p}$, we obtain the conclusion in this case.

Next assume that $h>t$, and hence $k>h$. We introduce $\mathbb{Z} \pi$-submodules of $M_{p}$

$$
\begin{aligned}
& M_{p}(i)=\left\{x \in M_{p} \mid p^{i} x=0\right\}, \\
& M_{p}(i, j)=M_{p}(i) /\left\{p^{j} M_{p} \cap M_{p}(i)\right\},
\end{aligned}
$$

and submodules of $F_{p}$

$$
\begin{aligned}
& F_{p}(i)=\left\{x \in F_{p} \mid p^{i} x=0\right\}, \\
& F_{p}(i, j)=F_{p}(i) /\left\{p^{j} F_{p} \cap F_{p}(i)\right\} .
\end{aligned}
$$

Then by the hypothesis, we have $M_{p}(h)=M_{p}$.

We have that $\left\{\mathbb{Z} / p^{i} \mathbb{Z}\right\} \pi$-modules $M_{p}(i)$ and $M_{p}(i, j)$ are direct summands of $\mathbb{Z} \pi \otimes F_{p}(i)$ and $\mathbb{Z} \pi \otimes F_{p}(i, j)$.

Let us now consider the following commutative diagram:

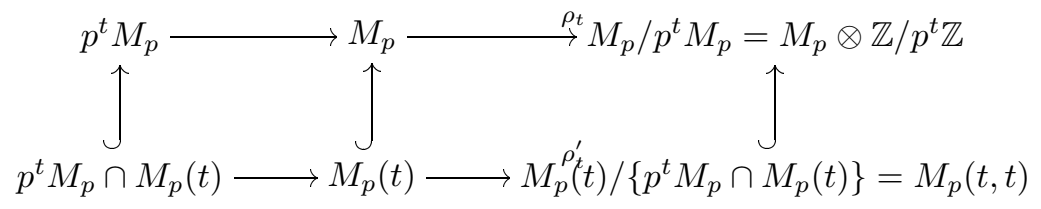


where $\rho_{t}$ and $\rho_{t}^{\prime}$ denote the canonical projections and all other lines denote the inclusions.

Let us recall that $k=2 h-t>h$ under the hypothesis. Since $F_{p}(t, t)$ is given as $F_{p}[t] \oplus\left\{p F_{p}[t+1]\right\} /\left(p^{t}\right) \oplus \ldots \oplus\left\{p^{h-t} F_{p}[h]\right\} /\left(p^{t}\right) \cong \sum_{i=\max (1,2 t-h)}^{t} F_{p}(t, t)[i]$, we have that $\operatorname{excess}\left(F_{p}(t, t)\right) \leq 2 t-(2 t-h)=h<k$ and hence, by the induction hypothesis, that $M_{p}(t, t)$ admits a direct sum decomposition $M_{p}(t, t) \cong \sum_{i=1}^{t} M_{p}(t, t)[i]$ as $\mathbb{Z} \pi$-modules, where $M_{p}(t, t)[i]$ is a free $\left\{\mathbb{Z} / p^{i} \mathbb{Z}\right\} \pi$-module and hence an induced $\mathbb{Z} \pi$-module from a free $\mathbb{Z} / p^{i} \mathbb{Z}$-module.

Since $M_{p}(t)$ is also a $\left\{\mathbb{Z} / p^{t} \mathbb{Z}\right\} \pi$-module, the canonical projection from $M_{p}(t)$ to $M_{p}(t, t)[t]$ has a splitting $s_{t}: M_{p}(t, t)[t] \rightarrow M_{p}(t)$.

Let us define $M_{p}[t]$ as the image $s_{t}\left(M_{p}(t, t)[t]\right) \subseteq M_{p}(t) \subseteq M_{p}$, which is an induced $\mathbb{Z} \pi$-module from a free $\mathbb{Z} / p^{t} \mathbb{Z}$-module. Then the image $\rho_{t}\left(M_{p}[t]\right)$ is nothing but $M_{p}(t, t)[t]$ in $M_{p}(t, t) \subseteq M_{p} / p^{t} M_{p}$, since $M_{p}[t]$ is in $M_{p}(t)$ and the restriction of $\rho_{t}$ to $M_{p}(t)$ coincides with $\rho_{t}^{\prime}: M_{p}(t) \rightarrow M_{p}(t, t)$. This implies that $M_{p}[t]$ is a direct summand of $M_{p}$.

Thus we obtain a direct sum decomposition $M_{p} \cong M_{p}[t] \oplus M_{p}^{\prime}$ as $\mathbb{Z} \pi$-modules, where $M_{p}^{\prime}$ is the kernel of the projection from $M_{p}$ to $M_{p}[t]$. On the other hand, we have a direct sum decomposition $F_{p}(h) \cong F_{p}[t] \oplus F_{p}^{\prime}$, where $F_{p}^{\prime}=F_{p}[t+1] \oplus \ldots \oplus F_{p}[h]$.

By the definition of $M_{p}[t]$, every generator in $M_{p}^{\prime}$ has the order $\geq p^{t+1}$, because a generator with the order $p^{t}$ has non-zero projection in $M_{p}[t]$.

Let us recall that $M_{p}$ is a direct summand of $\mathbb{Z} \pi \otimes F_{p}$, and hence there is a retraction from $\mathbb{Z} \pi \otimes F_{p}$ to $M_{p}^{\prime}$. Since an element in $F_{p}[t]$ has the order $<p^{t+1}$, the retraction restricted to $\mathbb{Z} \pi \otimes F_{p}[t]$ is trivial after reduced modulo $p$. Hence the retraction restricted to $\mathbb{Z} \pi \otimes F_{p}^{\prime}$ has to be a surjection after reduced modulo $p$, and hence is a surjection before reducing modulo $p$. Thus $M_{p}^{\prime}$ is a direct summand of $\mathbb{Z} \pi \otimes F_{p}^{\prime}$, where $\operatorname{excess}\left(F_{p}^{\prime}\right) \leq h-1+h-t=k-1<k$.

By the induction hypothesis, we have a direct sum decomposition of $M_{p}^{\prime}$ :

$$
M_{p}^{\prime} \cong M_{p}[t+1] \oplus M_{p}[t+2] \oplus \ldots \oplus M_{p}[h], \text { as } \mathbb{Z} \pi \text {-modules, }
$$

where $M_{p}[i]$ is an induced $\mathbb{Z} \pi$-module from a free $\mathbb{Z} / p^{i} \mathbb{Z}$-module for $t+1 \leq i \leq h$. Thus we obtain a direct sum decomposition of $M_{p}: M_{p} \cong M_{p}[t] \oplus M_{p}[t+1] \oplus \ldots \oplus$ $M_{p}[h]$ as desired. This completes the proof of the proposition.

Now we prove Lemma 4.3.

Proof. Let us assume that $M$ is a direct summand of $\mathbb{Z} \pi \otimes F, F$ a $\mathbb{Z}$-module. Then $M /$ torsion is a direct summand of $\mathbb{Z} \pi \otimes\{F /$ torsion $\}$.

Since $F /$ torsion is a free $\mathbb{Z}$-module, the free part of $M$ is a projective $\mathbb{Z} \pi$-module, and hence is free by Bass [1]. Thus we have the following isomorphism of $\mathbb{Z} \pi$ modules:

$$
M / \text { torsion } \cong \mathbb{Z} \pi \otimes M_{0}, M_{0} \text { is a free } \mathbb{Z} \text {-module. }
$$

To proceed, let us consider the $p$-torsion part $M_{p}$ and $F_{p}$ of $M$ and $F$, respectively:

$$
\begin{aligned}
& M \cong M_{0} \oplus \sum_{p: \text { all primes }} M_{p}, \\
& F \cong F_{0} \oplus \sum_{p: \text { all primes }} F_{p} .
\end{aligned}
$$


Since $F_{p}$ is finitely generated, $M_{p}$ satisfies the hypothesis of Proposition 4.5. Thus $M_{p}$ is also an induced $\mathbb{Z} \pi$-module from a finitely generated $p$-torsion module.

\section{The Proof of Corollary 2.2}

Let us assume that $B$ is a subspace of $X$. Then $\pi_{q}(X, B)$ is isomorphic with $\pi_{q}(\tilde{X}, \tilde{B})$. By the assumption, the Hurewicz homomorphism $\pi_{n}(\tilde{X}, \tilde{B}) \rightarrow H_{n}(\tilde{X}, \tilde{B})$ $=H_{n}(\tilde{X})$ is an isomorphism. Hence by Theorem $2.1, \pi_{n}(X, B) \cong \mathbb{Z} \pi \otimes H_{n}(X)$, where $H_{n}(X)$ can be described as a direct sum of cyclic groups $\Sigma_{a} \mathbb{Z} e_{a} \oplus \Sigma_{b} \mathbb{Z} / m_{b} \mathbb{Z} f_{b}$. Let $\alpha_{a}: S^{n} \rightarrow X$ and $\beta_{b}: S^{n} \rightarrow X$ be mappings corresponding to $e_{a}$ and $f_{b}$.

Then $m_{b} f_{b}=0$ implies that $\beta_{b}$ is extendable to the Moore space $S^{n}\left(m_{b}\right)=$ $S^{n} \cup_{m_{b}} e^{n+1}$, say $\gamma_{b}: S^{n}\left(m_{b}\right) \rightarrow X$. Hence we have a mapping $\phi_{1}: X_{n}=B \vee$ $\bigvee_{a} S^{n}{ }_{a} \vee \bigvee_{b} S^{n}\left(m_{b}\right) \rightarrow X$, which induces clearly an isomorphism of homology groups of the universal coverings up to dimension $n$. Thus $\phi_{1}$ is $n$-connective.

Let us assume that $X_{n}$ is a subspace of $X$. Then $\pi_{q}\left(X, X_{n}\right)$ is isomorphic with $\pi_{q}\left(\tilde{X}, \tilde{X}_{n}\right)$. By the assumption, the Hurewicz homomorphism $\pi_{n+1}\left(\tilde{X}, \tilde{X}_{n}\right) \rightarrow$ $H_{n+1}\left(\tilde{X}, \tilde{X}_{n}\right)=H_{n+1}(\tilde{X})$ is an isomorphism. Hence by Theorem $2.1, \pi_{n+1}\left(X, X_{n}\right)$ $\cong \mathbb{Z} \pi \otimes H_{n+1}(X)$, where $H_{n+1}(X)$ can be described as a direct sum of cyclic groups $\Sigma_{a^{\prime}} \mathbb{Z} e_{a^{\prime}}^{\prime}$. Let $\alpha_{a^{\prime}}^{\prime} \in \pi_{n+1}\left(X, X_{n}\right)$ be mappings corresponding to $e_{a^{\prime}}^{\prime}$.

Let us consider the following exact sequence:

$$
\cdots \rightarrow \pi_{n+1}(X) \rightarrow \pi_{n+1}\left(X, X_{n}\right) \stackrel{\partial}{\rightarrow} \pi_{n}\left(X_{n}\right) \rightarrow \pi_{n}(X) \rightarrow \cdots
$$

Here, by the theorem of Hurewicz, both the modules $\pi_{n}\left(X_{n}\right)$ and $\pi_{n}(X)$ are isomorphic with $H_{n}(\tilde{X})$, and hence, the above homomorphism $\partial$ is trivial. So, we may assume that $\alpha_{a^{\prime}}^{\prime}$ is a mapping from $S^{n+1}$ to $X$.

Hence we have a mapping

$$
\phi_{2}: X_{n+1}=X_{n} \vee \bigvee_{a^{\prime}} S^{n+1}{ }_{a^{\prime}}=B \vee \bigvee_{a} S_{a}^{n} \vee \bigvee_{b} S^{n}\left(m_{b}\right) \vee \bigvee_{a^{\prime}} S^{n+1}{ }_{a^{\prime}} \rightarrow X,
$$

which induces a homology equivalence of the universal coverings. Thus $\phi_{2}$ is a homotopy equivalence, which completes the proof of the corollary.

\section{REFERENCES}

1. H. Bass, Projective modules over free groups are free, J. of Algebra 1 (1964) 367-373. MR 31:2290

2. I. Berstein and E. Dror, On the homotopy type of non-simply connected co-H-space, Ill. Jour. Math. 20 (1976) 528-534. MR 53:11607

3. P. M. Cohn, Free ideal rings, J. of Algebra 1 (1964) 47-69. MR 28:5095

4. R. H. Crowell and R. H. Fox, Introduction to knot theory, Blaisdell Publishing Company, New York (1963). MR 26:4348

5. S. Eilenberg and T. Ganea, On the Lusternik-Schnirelmann category of abstract groups, Ann. of Math. 65 (1957) 517-518. MR 19, 52d

6. R. H. Fox, Free differential calculus I, Ann. of Math. 57 (1953) 547-560. MR 14, 843d

7. T. Ganea, Cogroups and suspensions, Invent. Math. 9 (1970) 185-197. MR 42:2484

8. T. Ganea, Some problems on numerical homotopy invariants, Lecture Notes in Math. 249, Springer-Verlag, Berlin (1971) 13-22. MR 49:3910

9. P. Hilton, G. Mislin and J. Roitberg, On co-H-spaces, Comment. Math. Helv. 53 (1978) 1-14. MR 80d: 55014

10. D. M. Kan, On monoids and their dual, Bol. Soc. Math. Mex. 3 (1958) 52-61. MR 22:1900

11. K. Komatsu, A boundary link is trivial if the Lusternik-Schnirelmann category of its complement is one, Osaka J. Math. 29 (1992) 329-337. MR 94d:57019 
12. N. Oda, Pairings and copairings in the category of topological spaces, Publ. RIMS Kyoto Univ. 28 (1992) 83-97. MR 93c:55013

13. S. Saito, Notes on Co-H-spaces, J. Fac. Sci. Shinshu Univ. 6 (1971) 101-106. MR 47:5867

14. On higher coassociativity, Hiroshima Math. J. 6 (1976) 589-617. MR 55:9078

15. C. S. Seshadri, Triviality of vector bundles over the affine space $K^{2}$, Proc. Natl. Acad. Sci. U.S. 44 (1958) 456-458. MR 21:1318

16. G. W. Whitehead, Elements of Homotopy Theory, GTM 61, Springer-Verlag, Berlin (1978). MR 80b:55001

Graduate School of Mathematics, Kyushu University, Ropponmatsu 4-2-1, Fukuoka 810, JAPAN

Current address: Department of Mathematical Sciences, University of Aberdeen, Aberdeen AB24 3QY, United Kingdom

E-mail address: iwase@math.kyushu-u.ac.jp, n.iwase@maths.abdn.ac.uk

Department of Mathematics, Shinshu University, Asahi 3-1-1, Matsumoto 390, Japan

Department of Art and Information Design, Kyushu Institute of Design, Shiobaru 4-9-1, FUKUOKA 815, JAPAN

E-mail address: sumi@kyushu-id.ac.jp 